\title{
Simultaneous Sensing cum Actuating Linear Motor
}

\author{
Ali Karimi Varkani \\ Tarbiat Modares University \\ Tehran, Iran \\ ali.karimi@modares.ac.ir
}

\author{
Ali Daraeepour \\ Duke University \\ Durham, North Carolina, USA \\ a.daraeepour@duke.edu
}

\begin{abstract}
This work aims to build up on a new measurement method to monitor the speed, velocity, and mechanical impedance of an electric machine (a linear motor) without using conventional sensors. Tested is a back-drivable linear motor stage which uses the linear motor electric machine as simultaneous sensor cum actuator. By calibrating the Transduction Matrix of the linear motor, the sensor cum actuator determines the velocity, load, and mechanical impedance of the motor by measuring the motor's time variant electrical impedance. The method is validated by an experimental procedure.
\end{abstract}

Index Terms-Sensor, Actuator, Electrical Motor, Monitoring, Measurement

\section{I.INTRODUCTION}

As the end use devices, electric machines, consume around $70 \%$ of the total electric power in the world hence their significance for global energy and sustainability is a top priority. More interestingly, a vital indicator of economic health is the growth in production and use of electric machinery applications. Today, the use of electric machines have proceeded far beyond the traditional paradigm of their basic application in production-lines that grew exponentially 2-3 generations ago. As of now personal computers, portable disk drives, advanced permanent magnet servos, and piezoelectric haptic actuators have created a new paradigm for developing, designing, and using the electric machines. Novelties that still continuously take place in this field have led to innovations in medical equipment, wind generation, aircraft systems, and numerous other applications. That being said, condition monitoring of the electric machines has been of tremendous interest to the industry. Specially, researchers have looked and are still looking for cost-effective yet reliable monitoring solutions. This paper tries to propose a method to overcome this challenge in specific case of a linear electric machine.

Back to the very basic concept, an electrical motor is a device that converts electrical power into mechanical power. Consider a motor as a system, its electrical impedance determines how much electric power it consumes. Hence monitoring impedance is important since it reveals if the energy is consumed in a healthy manner. Although there is substantial knowledge about electrical impedance, little is known about mechanical impedance. Similar to the electrical impedance which is defined by dividing the voltage by the current of the system, the mechanical impedance is defined by dividing the force by the velocity of the system.

Impedance control (i.e. mechanical impedance), proposed by Hogan [1], is usually employed in robotics and automatic manipulation of objects. The implementation of the control scheme requires installing force and velocity sensors. This adds to the intricacy and the finished cost of systems. Anderson et al [2] proposed a method to measure the deformation of a piezoelectric-coated cantilever beam by means of electromechanical modeling of the piezoelectric actuator. The simultaneous actuation and sensing was able to effectively predict and control the oscillation of the cantilever beam. The drawback to the method was that the loading effect 